\title{
Pavlovian inhibition cannot be obtained by posttraining A-US pairings: Further evidence for the empirical asymmetry of the comparator hypothesis
}

\author{
NICHOLAS J. GRAHAME, ROBERT C. BARNET, and RALPH R. MILLER \\ State University of New York, Binghamton, New York
}

\begin{abstract}
Water-deprived rats served in a conditioned lick suppression experiment to examine a prediction of the comparator hypothesis (Miller \& Matzel, 1988). Subjects received treatment analogous to Pavlovian conditioned-inhibition training, but without reinforcement (i.e., $\mathrm{A}-/ \mathrm{AX}-$ ). They then received reinforcement, that is, inflation, of Stimulus A (i.e., $A+$ ). According to the comparator hypothesis, this should have transformed Stimulus $\mathrm{X}$ into a conditioned inhibitor, especially under circumstances in which $A$ and $X$ are well associated (as might be expected following simultaneous compound preexposure to Stimuli $\mathrm{A}$ and $\mathrm{X}$ ). This experiment differed from previous demonstrations that showed that inflation effects could not be obtained, in that here, parameters were chosen both to promote a strong association between the target stimulus (i.e., Stimulus X) and its "comparator stimulus" (i.e., Stimulus A), and to minimize attenuation of this association during posttraining inflation of Stimulus A. No conditioned inhibition was observed relative to control groups that received inflation of an irrelevant stimulus (i.e., $\mathrm{A}-/ \mathrm{AX}-\rightarrow \mathrm{B}+$ ) or no simultaneous compound (i.e., $\mathrm{A}-/ \mathrm{X}-\rightarrow \mathrm{A}+$ ) exposure.
\end{abstract}

The comparator hypothesis of conditioned responding (Miller \& Matzel, 1988) posits that responding to a conditioned stimulus (CS) is inversely related to the current associative strength of other stimuli (i.e., comparator stimuli) that were present during training. To the extent that a CS has greater associative strength than its comparator stimulus, excitatory responding is expected. To the extent that the associative strength of a CS is weak relative to its comparator stimulus, inhibitory responding is expected. Posttraining extinction of comparator stimuli (i.e., deflation) has been found to increase excitatory responding or attenuate inhibitory responding over a wide range of preparations (e.g., Grahame, Barnet, \& Miller, in press; Matzel, Schactman, \& Miller, 1985). However, increasing the associative strength of comparator stimuli following training (also known as inflation) has not been found to affect responding to the target CS (Miller, Hallam, \& Grahame, 1990).

One reason why inflation of comparator stimuli may have little impact on responding to a CS is that the operation of inflating the comparator stimulus may have the effect of extinguishing the association between the target $\mathrm{CS}$ and the comparator stimulus. According to the comparator hypothesis, the association between a CS and its

Support for this research was provided by NIMH Grant 33881 and the SUNY-Binghamton Center for Cognitive and Psycholinguistic Sciences. We thank James Esposito for his services in collecting the data. Inquiries regarding this research should be addressed to $\mathbf{R}$. R. Miller, Department of Psychology, SUNY-Binghamton, Binghamton, NY 13902-6000. comparator stimulus is necessary because a CS must be able to evoke the representation of the comparator stimulus during testing in order for the comparator stimulus to affect responding to the CS. If the association between a CS and its comparator stimulus is extinguished as an unintended result of inflation training, the increased associative strength of the comparator stimulus would not be able to affect responding to the CS.

Operationally, inflation procedures might well extinguish the associations between a target CS and its comparator stimulus. Usually, inflation experiments have two phases. In the pretraining phase, compound presentations of the target CS and its comparator stimulus are presented without reinforcement. This is expected to result in the formation of an association between the CS and the comparator stimulus. In the inflation phase, the putative comparator stimulus is repeatedly paired with the unconditioned stimulus (US) in the absence of the target CS. For example, in all six experiments presented in Miller et al. (1990), inflation treatment consisted of a presentation of the putative comparator stimulus (either a context or a discrete cue) paired with a US anywhere from 12 to 176 times, depending on the specific experiment. Such extensive exposure to the comparator stimulus in the absence of the target CS might result in the extinction of the association between the target CS and its comparator stimulus.

In the present experiment, we attempt to obtain inflation effects using a procedure designed to minimize extinction between the target CS and its comparator stimulus (see Figure 1). Two relevant parameters were manip- 


\begin{tabular}{|c|c|c|c|}
\hline GROUP & TRAIN & INFLATION & TEST \\
\hline$w / C$ & $A-/ X-$ & At & $\mathrm{C} \longrightarrow$ ? \\
\hline$w / c x$ & $A-/ X-$ & At & $c x \rightarrow ?$ \\
\hline INF/C & $A-/ A X-$ & At & $c \longrightarrow$ ? \\
\hline INF/CX & $A-/ A X-$ & $A+$ & $c x \rightarrow ?$ \\
\hline NONINF/C & $A-/ A X-$ & $D+$ & $c \longrightarrow$ ? \\
\hline NONINF/CX & $A-/ A X-$ & $D+$ & $c x \rightarrow ?$ \\
\hline
\end{tabular}

Figure 1. Procedure of the experiment. / indicates alternates with; + indicates footshock; and $\mathrm{A}, \mathrm{X}, \mathrm{D}$, and $\mathrm{C}$ are four discriminable stimuli. See text for details.

ulated to obtain a strong CS-comparator stimulus association in experimental inflation groups (Groups INF/C and INF/CX). First, during the pretraining phase, we used a Pavlovian conditioned inhibition-like procedure, in which no reinforcers were given (i.e., $\mathrm{A}-/ \mathrm{AX}-$ ). This was expected to result in the formation of a robust association between Stimulus A (the comparator stimulus) and $X$ (the target CS) that would be resistant to extinction due to the partial "reinforcement" of Stimulus A with Stimulus $X$. In other words, subjects would be accustomed to receiving $\mathrm{A}$ without $\mathrm{X}$, such that there might be slower extinction of the $A-X$ association during the inflation phase, in which A would be presented with the US and without $X$. Second, there was only one A-US presentation during the inflation phase, which was expected to minimize extinction of the $\mathrm{A}-\mathrm{X}$ association.

We hoped that by using these procedures, we would be able to cause Stimulus $X$ to become a conditioned inhibitor, as measured by a summation test. Control subjects (Groups W/C and W/CX) would receive a pretraining phase in which $A$ and $X$ would not be paired (i.e., $\mathrm{A}-/ \mathrm{X}-)$, thereby precluding the formation of a withincompound $\mathrm{A}-\mathrm{X}$ association. Additional controls (Groups NONINF/C and NONINF/CX) received A-/AX - treatment followed by a noninflation treatment (i.e., $D+$ instead of $\mathrm{A}+$ ) during the inflation phase, which would not be expected to increase the associative strength of Stimulus X's comparator stimulus. Finally, all subjects received a summation test in a neutral test context with a known excitor other than that used in training (i.e., $\mathrm{C}$ vs. CX). If the training received by INF subjects resulted in Stimulus $\mathrm{X}$ becoming a conditioned inhibitor, we would expect more negative summation in INF subjects than in either W or NONINF subjects.

\section{METHOD}

\section{Subjects}

The subjects were 24 male and 24 female naive Sprague-Dawley rats from our own breeding colony. Weight ranges were 280-420 g for males and 190-300 $\mathrm{g}$ for females. Subjects were individually housed in wire-mesh cages in a vivarium maintained on a 16:8-h light:dark cycle. Running occurred approximately midway through the light phase. Subjects were allowed ad-lib access to food (Purina Lab Chow) in their home cages. A progressive water- deprivation schedule was administered over the week that running began, until water availability was limited to $10 \mathrm{~min}$ per day, given approximately $1 \mathrm{~h}$ after any scheduled treatment. Subjects were assigned to six groups $(n=8)$, counterbalanced for sex.

\section{Apparatus}

Two types of enclosures were used, counterbalanced as to which enclosure served as the training context (Context Train) and which enclosure served as the testing context (Context Test). Enclosure $\mathrm{X}$ was a clear, Plexiglas chamber in the shape of a box, 22.75 (length) $\times 8.25$ (width) $\times 13.0$ (height) $\mathrm{cm}$, with a floor constructed of 0.48 -cm-diam rods, $1.5 \mathrm{~cm}$ center-to-center and shorted via NE-2 neons, which allowed constant-current footshock to be delivered by means of a high-voltage ac circuit in series with a 1.0-M $\Omega$ resistor. Each of six copies of Enclosure $X$ had its own environmental isolation chest. Enclosure $Y$ could be brightly illuminated by a flashing ( $0.5 \mathrm{sec}$ on/ $0.5 \mathrm{sec}$ off) $25-\mathrm{W}$ (nominal at $120 \mathrm{~V} \mathrm{ac}$ ) bulb, driven at $100 \mathrm{~V}$ ac. When this cue light was not flashing, the enclosure was dimly illuminated by a $2.0-\mathrm{W}$ (nominal at $120 \mathrm{~V}$ ac) houselight, driven at $56 \mathrm{~V}$ ac. The flashing light was mounted on an inside wall of the environmental isolation chest, approximately $30 \mathrm{~cm}$ from the animal enclosure. Background noise (mostly from a ventilation fan) was $74 \mathrm{~dB}(\mathrm{C})$ re SPL. Each chamber was equipped with water-filled lick tube that extended $1 \mathrm{~cm}$ from the back of a cylindrical cull, $4.5 \mathrm{~cm}$ in diameter, and left-right centered, with its bottom $1.75 \mathrm{~cm}$ above the floor of the apparatus and $5.0 \mathrm{~cm}$ deep. There was a photobeam detector $1 \mathrm{~cm}$ in front of the lick tube. Three $45-\Omega$ speakers mounted on the interior sides of each chamber could deliver six clicks per second at an intensity of $8 \mathrm{~dB}(C)$ above the ambient background sound, a white noise of $10 \mathrm{~dB}(\mathrm{C})$ above background, and a complex tone $(3000$ and $3200 \mathrm{~Hz}) 8 \mathrm{~dB}(\mathrm{C})$ above background.

Enclosure $Y$ was a $25.5-\mathrm{cm}$-long box in a truncated-V shape ( $28 \mathrm{~cm}$ high, $21 \mathrm{~cm}$ wide at the top, $5.25 \mathrm{~cm}$ wide at the bottom). Each of six copies of Enclosure $Y$ had its own environmental isolation chest. The floor and sides were constructed of sheet metal and the ceiling was clear Plexiglas. The floor consisted of two parallel metal plates, each $2 \mathrm{~cm}$ wide, with a $1.25-\mathrm{cm}$ gap between them. Enclosure $Y$ could be brightly illuminated by a flashing ( $0.5 \mathrm{sec}$ on/0.5 sec off) 100 -W bulb (nominal at $120 \mathrm{~V} \mathrm{ac}$ ), driven at $100 \mathrm{~V}$ ac. When this cuelight was not flashing, the enclosure was dimly illuminated by a $7.5-\mathrm{W}$ (nominal at $120 \mathrm{~V}$ ac) houselight, driven at $56 \mathrm{~V}$ ac. The flashing light was mounted on an inside wall of the environmental isolation chest, approximately $30 \mathrm{~cm}$ from the animal enclosure. Light entered the animal enclosure primarily by reflection off the roof of the environmental chest. Background noise (mostly from the ventilation fan) was $74 \mathrm{~dB}(\mathrm{C})$. Each chamber was equipped with a water-filled lick tube that extended $1 \mathrm{~cm}$ from the back of a cylindrical cull, $4.5 \mathrm{~cm}$ in diameter, and left-right centered, with its bottom $5.5 \mathrm{~cm}$ above the floor of the apparatus and $5.0 \mathrm{~cm}$ deep. There was a photobeam detector $1 \mathrm{~cm}$ in front of the lick tube. Three $45-\Omega$ speakers mounted on the interior sides of each chamber could deliver six clicks per second at an intensity approximately $8 \mathrm{~dB}(\mathrm{C})$ above the ambient background sound, a white noise of $10 \mathrm{~dB}(\mathrm{C})$ above background, and a complex tone $(3000$ and $3200 \mathrm{~Hz}) 8 \mathrm{~dB}(\mathrm{C})$ above background.

In both of the above Enclosures, Stimuli $A$ and $D$ were counterbalanced within groups between the complex tone and the light. Stimulus $\mathrm{X}$ was the white noise for all subjects.

In addition, another type of chamber, designated as Context Long (of which there were 12 copies), was used for training the transfer excitor (clicks) to be used in the summation testing. These chambers measured 51 (length) $\times 16.5$ (width) $\times 75$ (height) $\mathrm{cm}$, and had end walls constructed of opaque green Plexiglas and long walls of aluminum. The floor was made of stainless steel rods, $0.64 \mathrm{~cm}$ in diameter $(1.8 \mathrm{~cm}$ center-to-center) positioned lengthwise in the 
chambers and shorted together with NE-2H neon bulbs in series with a 1-M $\Omega$ resistor to produce a constant-current, 2.0-mA footshock. No lick tubes were present. These chambers were housed in a well-lighted (120-W of fluorescent illumination) experimental room. These chambers were not in environmental isolation chests.

\section{Procedure}

Shaping in Context Test (Day 1). All subjects were placed in Context Test for a 60-min session, during which lick tubes were available. Latencies to complete the first and second 5 cumulative seconds of licking were recorded.

Shaping in Context Train (Day 2). All subjects were placed in Context Train for a 60 -min session, during which lick tubes were available. Latencies to complete the first and second 5 cumulative seconds of licking were recorded.

Pretraining phase (Days 3, 4, 7, 8, and 9). The subjects were placed in Context Train for 1-h daily sessions. The subjects in Groups INF/C, INF/CX, NONINF/C, and NONINF/CX received four nonreinforced presentations of $\mathrm{A}$, and eight nonreinforced simultaneous compound presentations of both $A$ and $X$. Groups W/C and $W / C X$ received four nonreinforced presentations of $A$, and eight nonreinforced presentations of $\mathrm{X}$ alone. All stimulus durations were $30 \mathrm{sec}$.

Known excitor training (Days 5-6). The subjects were placed in Context Long for 1-h daily sessions. They received four $15-\mathrm{sec}$ presentations of the clicks, each reinforced with a 2-mA footshock during the last $2 \mathrm{sec}$ of the clicks.

Inflation training (Day 10). All subjects were placed in Context Train for a 1-h session. Groups INF/C, INF/CX, W/C, and W/CX received three pairings of Stimulus $A$, terminating with a 2-mA, 2-sec footshock US. Groups NONINF/C and NONINF/CX received three presentations of Stimulus $D$, terminating with the same footshock. Trials occurred at 5, 20, and $35 \mathrm{~min}$ into the session.

Reshaping in Context Test (Days 11 and 12). To reestablish baseline lick rates, all subjects received 60 -min daily sessions with no nominal CSs or USs presented. Latencies to complete the first 5 cumulative seconds of licking were recorded. Any subjects that failed to complete 5 cumulative seconds of licking within $60 \mathrm{sec}$ after being placed in their chambers on Day 12 were subjected to an additional 30-min reshaping session 1-2 $\mathrm{h}$ after the first reshaping session of that day. This was to ensure equal baseline licking on the Day 13 CS test. Seven subjects were given this additional treatment ( 2 in Group INF/CX, 2 in Group INF/C, 2 in Group W/CX, and 1 in Group W/C).

Summation Test 1 (Day 13). Within Context Test, the subjects in $\mathrm{C}$ groups were tested on the clicks alone, while the subjects in CX groups were tested on a simultaneous compound of the clicks and Stimulus $X$ in a 16-min test. The appropriate test-stimulus onset immediately followed the completion of 5 cumulative seconds of drinking, and remained on for the next $15 \mathrm{~min}$ of the test, thereby ensuring equal exposure to the test stimulus in all animals. Latencies to complete the first 5 cumulative seconds of licking (pre-CS) and the second 5 cumulative seconds of licking (in the presence of the CS) were recorded.

Reshape in Context Train (Day 14). All subjects were placed in Context Train for $1 \mathrm{~h}$ with no nominal CSs or USs presented. Latencies to complete the first and second 5 cumulative seconds of licking were recorded.

Test A (Day 15). All subjects were placed in Context Train. Stimulus A onset followed the completion of 5 cumulative seconds of drinking, and remained on until the subjects had completed the second 5 cumulative seconds of drinking in the presence of the CS. The animals were tested in Context Train because earlier experiments have demonstrated that the light CS, unlike the auditory CSs, does not generalize well between contexts.

\section{RESULTS}

No differences were seen between groups during the first 5 cumulative seconds of drinking, that is, prior to the onset of the test CS, on either Day 13 or Day 15 $(F \mathrm{~s}<1)$.

Suppression during the second 5 cumulative seconds of licking on Day 13, that is, during the summation test (C vs. CX; see Figure 2) was analyzed by a 3 (W, INF, or NONINF) $\times 2$ (C vs. CX) analysis of variance (ANOVA). This analysis indicated a main effect of test stimulus $[F(1,42)=2.53, p<.05]$, indicating less responding during the $\mathrm{CX}$ compound than during $\mathrm{C}$ alone. No other main effects or interactions approached significance (all Fs $<1.00$ ).

A one-way ANOVA (W, INF, or NONINF, see Figure 3) conducted on the Stimulus A test on Day 15 indicated a main effect of training group $[F(2,42)=3.83$, $p<.05]$. Planned comparisons indicated that this effect resulted from Group NONINF exhibiting less fear of Stimulus A than either Group W or Group INF $\left[F_{s}(1,42) \geq\right.$ $5.47, p s \leq .05$ ], consistent with its lack of inflation training with Stimulus $A$.

\section{DISCUSSION}

Stimulus $\mathrm{X}$ decreased suppression to the excitatory clicks in all three training conditions. The uniformity of this negative summation across all conditions suggests the presence of nonassociative, external inhibition, rather than any associative conditioned inhibition. A lack of conditioned inhibition in INF subjects indicates that an inflation effect was not obtained as a result of pairing the comparator stimulus with the US following AX preexposure.

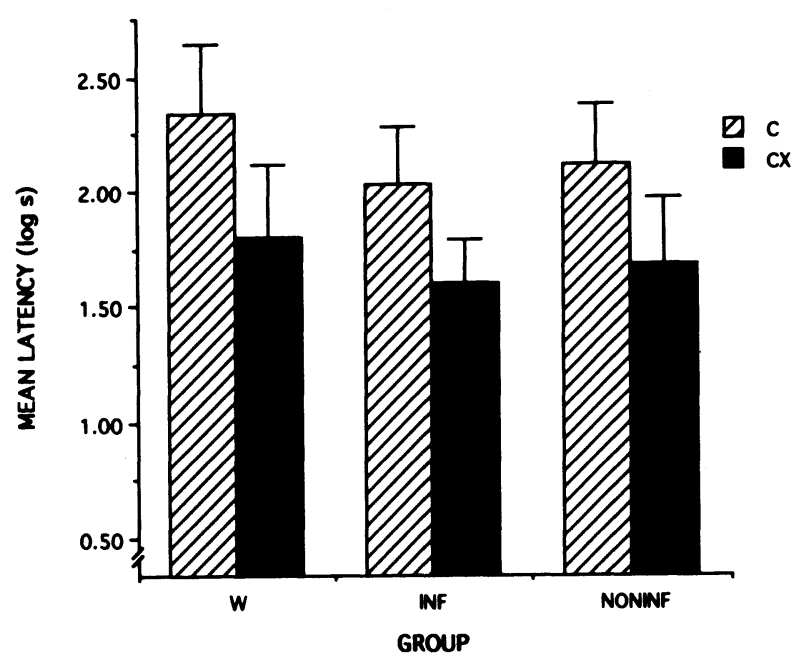

Figure 2. Results of the second 5 cumulative seconds of drinking in the presence of the CS during the summation test on Day 13. C indicates testing with the known excitor, that is, the clicks, and CX indicates the summation compound of the clicks and the putative inhibitor. See text for details of group treatments. Brackets indicate standard errors. 


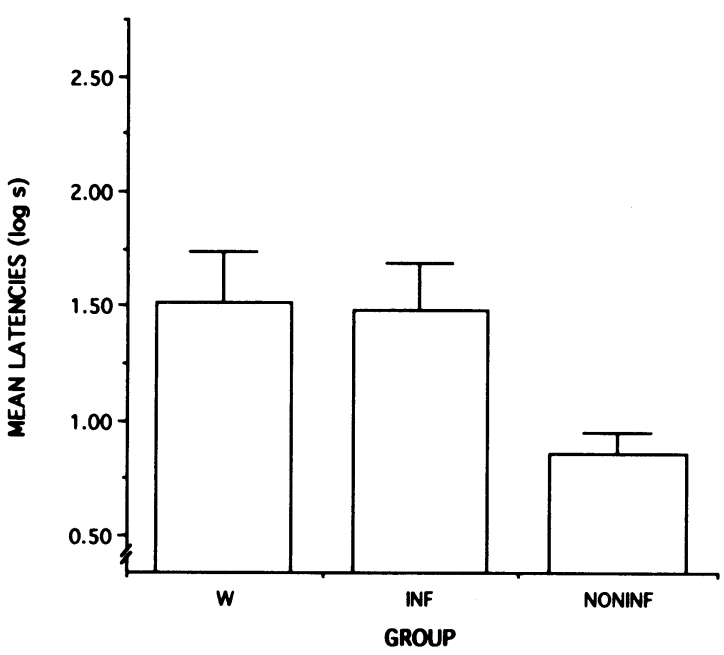

Figure 3. Results from Day 15 of the second 5 cumulative seconds of drinking, that is, in the presence of Stimulus A. See text for details of group treatments. Brackets indicate standard errors.

The failure to obtain effects on responding to the target CS of inflating the presumed comparator stimulus, when using parameters intended to maximize the CS-comparator stimulus association, suggests that extinction of CS-comparator stimulus associations probably does not account for the lack of inflation effects in other preparations. The present results emphasize the asymmetry between posttraining inflation and deflation of comparator stimuli in the ability of the compara- tor hypothesis to predict changes in responding to a target CS. Specifically, many experiments demonstrate the ability of posttraining extinction of comparator stimuli to increase excitatory responding (or decrease inhibitory responding) to a target CS. One way of accounting for this apparent asymmetry might have been to appeal to the divergent effects of posttraining inflation versus deflation procedures. Deflation experiments probably result in the extinction of both the CS-comparator stimulus association and the comparator stimulus-US association. In principle, the extinction of either association would result in an increase in the response potential of excitatory CSs. Conversely, inflation might result in the extinction of the CS-comparator stimulus association, which would obscure the effects of the acquisition of a comparator stimulus-US association. Based on the results of the present experiment, however, it does not appear that the extinction of the CS-comparator stimulus associations underlies the failure to obtain effects of inflation.

\section{REFERENCES}

Grahame, N. J., Barnet, R. C., \& Miller, R. R. (in press). Pavlovian conditioning in multiple contexts: Competition between contexts for comparator status. Animal Learning \& Behavior.

Matzel, L. D., Schachtman, T. R., \& Miller, R. R. (1985). Recovery of an overshadowed association achieved by extinction of the overshadowing stimulus. Learning \& Motivation, 16, 395-412.

Miller, R. R., Hallam, S. C., \& Grahame, N. J. (1990). Inflation of comparator stimuli following CS training. Animal Learning \& Behavior, 18, 434-443.

MilleR, R. R., \& MATZEL, L. D. (1988). The comparator hypothesis: A response rule for the expression of associations. In G. H. Bower (Ed.), The psychology of learning and motivation, (Vol. 22, pp. 51-92). Orlando, FL: Academic Press.

(Manuscript received April 23, 1992.) 